\title{
Analyzing Educational Objectives that Include Critical Thinking: Dot Product Problems in Vector Algebra
}

\author{
Aneta Gacovska-Barandovska \\ Ss. Cyril and Methodius University in Skopje, Arhimedova 3, 1000 Skopje, North Macedonia, \\ aneta@pmf.ukim.mk \\ Vesna Celakoska-Jordanova \\ Ss. Cyril and Methodius University in Skopje, Arhimedova 3, 1000 Skopje, North Macedonia \\ Emilija Celakoska \\ Ss. Cyril and Methodius University in Skopje, Karposh II, 1000 Skopje, North Macedonia
}

\begin{abstract}
The primary and secondary school educational system should be stable and any upgrading reforms should be made gradually and consistently. This is especially important in mathematics education, since the element of logical reasoning while learning is more prominent there. Inconsistencies in reforms generate deficiencies in the higher levels of young students' reasoning skills and this situation continues on the university stage of education. We will report our findings about the reasoning of first-year university students on elements of geometry and associated algebra. We conducted an experiment where students' understanding of the definition of dot product of two vectors, cosine function and linear (in)dependence of vectors is evaluated, and address their mathematical activity to provide insight into the key elements of the problem they are solving. We use Bloom's and SOLO taxonomy as a tool for the assessment of our findings. We obtained the data from written exams given to vector algebra students and also from individual interviews.
\end{abstract}

Keywords: Reasoning, Problem solving, Students' performance, Knowledge structural levels

\section{Introduction}

National assessments and other educational investigations have acknowledged that there is a constant need for improvement in mathematics teaching and learning. The basic idea of today's educational research is to contribute to improving learning with understanding. The constant changes made to our state educational system are not always comprehensive. In the authors' opinion, partially reforming separate stages of education leads to the fact that an overall effect in math achievements is disconnectedly adopted knowledge. So, when it comes to the application of mathematical skills which require in-depth understanding of interconnections within the learnt material, the observed results are unsatisfactory. It seems that the students' competence in mathematical applications developed in a shallow, superficial fashion. It brings us to the question, does our university produce good math teachers and are they prepared to respond to new challenges? How does a lack of previously adopted knowledge affect math teachers' preparedness and in what ways does it hamper their motivation for acquiring teaching skills? Is creating a good math student sufficient for having a good math teacher? These questions, along with identifying the reforms' missteps with respect to primary and secondary school mathematics, arise while working with university students. The observed gap between the obtained and expected level of students' performance in the first year of math studies, prompted us to check the fulfillment of some standard educational objectives amongst our students. Findings of this type should not be understood as a plain critique of students' skills, but as an investigation of the pathways in the system where the acquirement of the mathematical critical reasoning skills has weakened. We accept that mathematical problem solving skills and common critical thinking are related, (Alcantara and Bacsa 2017; Sumarna et al. 2017; Jacob 2012), etc., which makes mathematics a core academic skill for forming successful individuals, persons who can debate their ideas with others and who can advocate for their ideas in a constructive manner. These are traits of citizens in a highly functional society. We address the issue of critical thinking skills, in a group of 30 first-year university students that enrolled mathematics module at the Faculty of Natural Sciences and Mathematics in Skopje, in the winter semester of 2018/2019. We use Bloom's and SOLO taxonomy, as a theoretical basis, to evaluate the students' performance on three selected problems from a Vector algebra (VA) course in connection with the geometric definition of a dot product of two vectors. 
Why should we consider such a simple problem? Understanding the notion of dot product of two vectors as well as possible in the first year of studies (first semester) is very important, since it has many applications in linear algebra and physics. The notion of dot product of two vectors in three-dimensional Euclidean space can be generalized to inner product for vectors in higher dimensions in real and complex vector spaces by abstracting the properties of the dot product. Certainly, dot product is used in the higher course of Analytic geometry in the second semester, as well as applications in physics and the mentioned generalization is a topic of several other courses: Physics 1 (a course in the third semester) and second and sixth semester courses of Linear Algebra and Vector Spaces, respectively.

The need of this type of investigation has been underlined in 1906, when Henry S. White considered how the course Analytic Geometry should be taught. Although more than a century ago, some conclusions can easily be transferred to Vector Algebra and today's math teaching: the educational value of a subject is found less in its extension than in its intension; less in the multiplicity of its parts than in their unification through a few fundamental or climactic principles, (White 1906). The transfer of knowledge and full and complete utilization of previously adopted knowledge, in this sense, is a major educational benefit.

The obtained data from the conducted experiment indicate that students find it hard to adapt from the way of teaching in high school into the new way of teaching at the faculty. Even though they have only 10 subjects in the first year ( 5 in each semester), many of them cannot get used to the faster pace of teaching and to the requirement for consistency in learning the new material. This brings us to the following problems: 1) Can students relate to the material that is learned in high school? 2) Can they clearly understand the lecture notes and exercises, including newly introduced notions? Can they properly use the recommended literature? Are they willing to use other materials or resources when faced with difficulties in understanding? 3) Can they apply the obtained knowledge in a new situation? We will try to provide some answers to these questions, since we are confident that there are enough simple problems in the first year of mathematics university studies that can be used for the development of critical thinking that "should not wait until students take advanced-mathematics courses", (Harel and Sowder 2005).

\section{Theoretical Background}

What does critical thinking mean? A comprehensive review of the many approaches to this matter is given by (Lai 2011) and it includes: philosophical approach, which focuses on the qualities of an ideal thinker; the cognitive psychological approach that focuses on how people think in real situations, and also the educational approach that categorizes students' cognitive learning. The most cited work among educational practitioners on critical thinking is Bloom's taxonomy (classification) of educational objectives, (Bloom et al. 1956). By this classification, students' cognitive skills are ordered hierarchically, i.e. there are six stages of cognition: knowledge, comprehension, application, analysis, synthesis and evaluation. Each stage has a precise definition (see Table 1). Bloom's taxonomy model has been revised by (Anderson and Krathwohl 2001). The original six stages "were retained, but with important changes. Three categories were renamed, the order of two was interchanged and those category names retained were changed to verb form to fit the way they are used in objectives", (Krathwohl 2002) (see Table 1).

Critical thinking objectives for business curriculums are considered in (Zapalska et al. 2018). Here, in a similar way, we discuss the critical thinking skills that four years university students in mathematics are required to have. We have arranged them in the following way: students in the first-year level must develop skills such as remembering, understanding and applying; second-year students are not only expected to have the first three stages of skills, but to analyze the material and make relationships between concepts and ideas; by the end of the fourth-year level, students are expected to evaluate ideas, to make links between theories, or between theories and real world problems and to create something new (such as their graduate thesis or to write an essay on a popular mathematical subject.

The first stage of Bloom's taxonomy is very important for mathematics students. It can be described by the question "Can the student recall or remember the information?", (Zapalska et al. 2018). Firstly, one cannot stress enough how important it is for first-year mathematics students to have good background knowledge of elementary mathematics. They should be able to remember the high school mathematical terminology with ease, formulas, methods of solving geometry, trigonometry or algebra problems... Secondly, students must also remember the newly acquired concepts and ideas so that the analytical process may begin. "Without memorization, the critical thinking process cannot proceed further", (Zapalska et al. 2018). 
The second stage of understanding is essential in the first year of student academic education. It can be described with the question "Can the student explain concepts or ideas?", (Zapalska et al. 2018). In mathematics this means that the student must know the definitions of the notions and formulations of important theorems or propositions, as well as examples and counterexamples related to the subject. The first-year mathematics students must overcome the basic notions of mathematical logic, which will enable them to understand the ways of conducting proofs of theorems. By mastering this stage, the student can be certain that they will be successful in the future learning process.

The third stage of the educational process, applications, can be described with the question "Can the student use the information in a new way?", (Zapalska et al. 2018). This is the most difficult part for a mathematics student, where they are asked to identify the problem and to apply the learned notions, rules, methods and properties to a specific problem.

The fourth stage, analyzing, corresponds to the question "Can the student explain the relationships between different parts of the material?", (Zapalska et al. 2018). Students are expected to break down the material into constituent parts, to determine how these parts relate to one another or how they interrelate and then recognize how they are organized as a whole. In this higher stage of education, they should be able to recognize and explain patterns or relationships between different concepts they have learned. This stage is crucial for the students' independent thinking.

The fifth stage of the educational process is evaluating, i.e. making judgments about the value of ideas, methods, solutions, works, etc. Even though evaluation is at the end of this classification, it does not mean that it cannot lead to new analyses, acquiring new knowledge or discovering a new application, (Bloom et al. 1956). Also, some criteria in the evaluation process should be posted and followed (for instance, logical accuracy, consistency and absence of errors or the use of techniques, methods or rules). Either way, the student should be able to justify a position.

The sixth stage, creating, is the highest form of learning, since the student must be able to create something on their own (usually for undergraduate mathematics students this means a work on their graduate thesis or, in some cases, publishing a paper on a popular mathematical subject). Such tasks are highly absorbing (if done properly) and they can bring the student a great personal satisfaction, (Bloom et al. 1956).

Table 1. The Cognitive Process Dimension (Krathwohl 2002)

\begin{tabular}{|c|c|}
\hline Bloom's taxonomy 1956 & Anderson and Krathwohl's taxonomy 2001 \\
\hline Knowledge & Remember \\
\hline $\begin{array}{l}\text { Remembering of previously learned material (includes } \\
\text { recalling and repeating memorized facts, definitions, } \\
\text { methods, theories). }\end{array}$ & $\begin{array}{l}\text { Recognizing or recalling knowledge from } \\
\text { memory. Memory is used to produce or retrieve } \\
\text { definitions, facts, concepts, key ideas, etc. }\end{array}$ \\
\hline Comprehension & Understand \\
\hline $\begin{array}{l}\text { The ability to grasp or construct a meaning of } \\
\text { previously learned material. This may be demonstrated } \\
\text { by interpretation of the material (explaining, } \\
\text { differentiating or concluding). }\end{array}$ & $\begin{array}{l}\text { A clear understanding of material and constructing } \\
\text { meaning by interpreting, exemplifying, } \\
\text { classifying, summarizing, inferring, comparing or } \\
\text { explaining. }\end{array}$ \\
\hline Application & Apply \\
\hline $\begin{array}{l}\text { The ability to use learned material, or to } \\
\text { implement it in new and concrete situations. }\end{array}$ & $\begin{array}{l}\text { Carrying out or using a procedure in a given } \\
\text { situation. }\end{array}$ \\
\hline Analysis & Analyze \\
\hline $\begin{array}{l}\text { The ability to break down the material into parts and to } \\
\text { identify the relationship between them, so that its } \\
\text { organizational structure may be understood. }\end{array}$ & $\begin{array}{l}\text { Breaking material into its constituent parts } \\
\text { and detecting how the parts relate to one another } \\
\text { and to an overall structure or purpose. }\end{array}$ \\
\hline Synthesis & Evaluate \\
\hline $\begin{array}{l}\text { The ability to put parts together to form a coherent } \\
\text { or unique new whole. This means assembling, } \\
\text { combining and organizing part of the material to } \\
\text { prepare, formulate or construct new material. }\end{array}$ & $\begin{array}{l}\text { Making judgments based on criteria and } \\
\text { standards through checking and critiquing. } \\
\text { It is often a necessary part of the precursory } \\
\text { behavior before one creates something. }\end{array}$ \\
\hline Evaluation & Create \\
\hline $\begin{array}{l}\text { The ability to judge, check, estimate, argue and even } \\
\text { criticize the value of material for a given purpose. }\end{array}$ & $\begin{array}{l}\text { Putting elements together to form a novel, } \\
\text { coherent whole or make an original product. }\end{array}$ \\
\hline
\end{tabular}


When it comes to developing critical thinking among mathematics students, one cannot circumvent the SOLO taxonomy, since it provides a model for the levels of understanding. The abbreviation SOLO stands for Structure of the Observed Learning Outcome. This taxonomy proposed by (Biggs and Collis 1982; Biggs and Tang 2011) represents a systematic way of describing how students' performance grows in complexity when mastering tasks. There are five levels of understanding: pre-structural (the student is unsure of the lesson or the subject, uses irrelevant information and/or misses the point altogether), uni-structural (students know only the basic concepts of a subject), multi-structural (student approaches the subject in several aspects, but they are treated separately and are disconnected), relational (the complexity of the subject is mastered and the student can join all the parts together, i.e. the student can form a structure of the subject and therefore has competence to compare, relate, analyze and apply) and extended abstract (students' knowledge has reached a higher level, where they can look at the subject in a new way, can make abstractions and generalizations on a certain topic or in a certain area).

\section{Teaching Experiment in the Light of the First Three Stages of Bloom's Taxonomy}

We have chosen three dot product problems to investigate the first three stages of Bloom's educational classification, since they are essential for the beginning of the mathematical education of prospective mathematics teachers. The assignments were given during the last three weeks of the fall semester of 2018 on written tests. In this Section, the solutions to the problems and comments about the educational value of the proposed experimental problems are provided as well.

Each of these problems requires the student to transfer from a geometric description of the problem (where one uses the notions such as point, line or vector), to an algebraic description of the problem (where one uses linear combination, linear dependence of vectors or methods for solving systems of linear equations). In what follows, we discuss the students' responses and the difficulties they have in the first three stages of learning. We will evaluate the levels of understanding in each of the problems using the SOLO taxonomy.

The fundamental definition of a dot product (or scalar product) of two vectors states that it is the product of the lengths of each vector and the cosine of the angle between them, i.e. $\boldsymbol{a} \cdot \boldsymbol{b}=|\boldsymbol{a}| \cdot|\boldsymbol{b}| \cdot \cos \angle(\boldsymbol{a}, \boldsymbol{b})$.

The first thing that students learn from the lectures is that the dot product of two vectors results in a real number, not a vector. The next thing they are asked to notice is that if the angle between the vectors $\boldsymbol{a}$ and $\boldsymbol{b}$ is $\pi / 2$, i.e. if the vectors are perpendicular, then the dot product equals zero. And, if the angle is 0 or $\pi$, i.e. the vectors are collinear, the dot product coincides with \pm the product of the lengths of the vectors.

The first problem we considered requires the students to manage the definition of dot product, which is supposed to be well understood. From a mathematical perspective, the problem is procedural in nature. However, we expected the students to react when "something weird" appeared in the solution and use their knowledge on cosine function obtained in high school. It is also expected to search for the reasons why using the right techniques leads to a weird solution, if noticed. To our discontent, that was not the case.

Problem 1. Let vectors $\boldsymbol{a}=6 \boldsymbol{m}+2 \boldsymbol{n}$ and $\boldsymbol{b}=8 \boldsymbol{m}+3 \boldsymbol{n}$ be such that $|\boldsymbol{a}|=|\boldsymbol{b}|=1$ and the angle between the vectors $\boldsymbol{a}$ and $\boldsymbol{b}$ be $\angle(\boldsymbol{a}, \boldsymbol{b})=\frac{\pi}{3}$. Determine the angle between the vectors $\boldsymbol{m}$ and $\boldsymbol{n}$, if $|\boldsymbol{m}|=|\boldsymbol{n}|=3$.

Solution. From the given conditions and the definition of the dot product we obtain that $\boldsymbol{a} \cdot \boldsymbol{b}=|\boldsymbol{a}| \cdot|\boldsymbol{b}| \cdot \cos \angle(\boldsymbol{a}, \boldsymbol{b})=\cos \frac{\pi}{3}=\frac{1}{2}$. On the other hand,

$$
\boldsymbol{a} \cdot \boldsymbol{b}=(6 \boldsymbol{m}+2 \boldsymbol{n}) \cdot(8 \boldsymbol{m}+3 \boldsymbol{n})=48|\boldsymbol{m}|^{2}+34 \boldsymbol{m} \cdot \boldsymbol{n}+6|\boldsymbol{n}|^{2}=48 \cdot 9+34 \cdot 3 \cdot 3 \cdot \cos \angle(\boldsymbol{m}, \boldsymbol{n})+6 \cdot 9 .
$$

Comparing these two equations we conclude that $\frac{1}{2}=486+306 \cdot \cos \angle(\boldsymbol{m}, \boldsymbol{n})$ which implies that $\cos \angle(\boldsymbol{m}, \boldsymbol{n})=-1,587$. Considering the fact that $\cos \angle(\boldsymbol{m}, \boldsymbol{n}) \notin[-1,1]$, it is clear that vectors that satisfy the given conditions do not exist.

Most of the students got half way through the solution. None of the students that have nearly solved the problem noticed that the value of the cosine is beyond the range of the cosine function, and despite the technically correct 
procedure, none of them have presented a conclusion, a comment or a final answer. Not to mention, as perspective teachers, none of them even wondered why the answer is out of range, or if there are more than enough conditions given (although this question takes us to the higher stages of the classification of knowledge).

$$
\begin{aligned}
& 34 \bar{m} \bar{n}=\frac{1}{2}-432-54 \\
& 68 \bar{m} \bar{n}=1-864-108 \\
& 68 \bar{m} \bar{n}=-971 \\
& \bar{m} \bar{n}=-\frac{971}{68} \\
& \cos *(\bar{m}, \bar{n})=\frac{\bar{m} \cdot \bar{n}}{|\bar{m}|(\bar{n} \mid} \\
& \cos *(\bar{m}, \bar{n})=\frac{-\frac{971}{68}}{3.3}=\frac{-971}{68.9}=-\frac{971}{612}<-1
\end{aligned}
$$
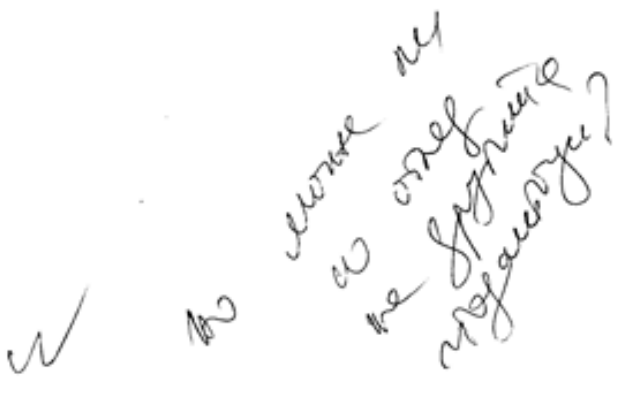

Figure 1. The Work of a Student whose Performance is Amongst the Few that Understood the Problem.

Only two students suspected that something was wrong with the conditions of the given problem, but did not confirm their suspicions (got half way through). Just looking at the lengths of the vectors $\boldsymbol{m}$ and $\boldsymbol{n}$, one can intuitively sense that something would "not add up" in the numerical solution of the problem. Namely, considering the lengths of the vectors $\boldsymbol{a}$ and $\boldsymbol{b}$, and the fact that $|\boldsymbol{a}|=|\boldsymbol{b}|=1$, we obtain that

$|\boldsymbol{a}|^{2}=(6 \boldsymbol{m}+2 \boldsymbol{n})^{2}=36|\boldsymbol{m}|^{2}+24 \boldsymbol{m} \cdot \boldsymbol{n}+4|\boldsymbol{n}|^{2}=360+24 \boldsymbol{m} \cdot \boldsymbol{n}$, i.e. $24 \boldsymbol{m} \cdot \boldsymbol{n}=-359$,

$|\boldsymbol{b}|^{2}=(8 \boldsymbol{m}+3 \boldsymbol{n})^{2}=64|\boldsymbol{m}|^{2}+48 \boldsymbol{m} \cdot \boldsymbol{n}+9|\boldsymbol{n}|^{2}=657+48 \boldsymbol{m} \cdot \boldsymbol{n}$, i.e. $48 \boldsymbol{m} \cdot \boldsymbol{n}=-656$.

Dividing this last equation by 2 , one obtains that $24 \boldsymbol{m} \cdot \boldsymbol{n}=-328$. This clearly contradicts the result $24 \boldsymbol{m} \cdot \boldsymbol{n}=-359$. This means that in the requirements of the problem there is no need of the vector $\boldsymbol{b}$ (neither its form as a linear combination nor its length). There is no need of the angle between the vectors $\boldsymbol{a}$ and $\boldsymbol{b}$, as well.

Namely, considering the fact that $24|\boldsymbol{m}| \cdot|\boldsymbol{n}| \cdot \cos \angle(\boldsymbol{m}, \boldsymbol{n})=-359$, one can verify that $\cos \angle(\boldsymbol{m}, \boldsymbol{n})=\frac{-359}{24|\boldsymbol{m}| \cdot|\boldsymbol{n}|}=\frac{-359}{24 \cdot 9}=-1,66$, is a contradiction, i.e. such vectors do not exist.

In what follows we will see a problem that is properly set. However, the students have difficulties in understanding the problem when formulated as it is.

Problem 2. (Samardžiski 1991) a) Determine a vector $\boldsymbol{n}$ that is coplanar with the vectors $\boldsymbol{p}$ and $\boldsymbol{q}$, if $|\boldsymbol{p}|=4$, $|\boldsymbol{q}|=1$, the angle between the vectors $\boldsymbol{p}$ and $\boldsymbol{q}$ is $\pi / 3$ and $\boldsymbol{n} \cdot \boldsymbol{p}=7, \boldsymbol{n} \cdot \boldsymbol{q}=3$.

b) Determine the length of the vector $\boldsymbol{n}$ and the angle between the vectors $\boldsymbol{n}$ and $\boldsymbol{p}$.

Solution. a) Since $\boldsymbol{n}$ is coplanar with the vectors $\boldsymbol{p}$ and $\boldsymbol{q}$ it follows that there are $\lambda, \mu \in \mathbf{R}$, such that $\boldsymbol{n}$ is a linear combination of the vectors $\boldsymbol{p}$ and $\boldsymbol{q}$, i.e. $\boldsymbol{n}=\lambda \boldsymbol{p}+\mu \boldsymbol{q}$. Multiplying this equality first by the vector $\boldsymbol{p}$ and then by the vector $\boldsymbol{q}$ we obtain the following:

$$
\begin{aligned}
& \text { 1) } \boldsymbol{n} \cdot \boldsymbol{p}=\lambda|\boldsymbol{p}|^{2}+\mu \boldsymbol{p} \cdot \boldsymbol{q} \Leftrightarrow \boldsymbol{n} \cdot \boldsymbol{p}=\lambda|\boldsymbol{p}|^{2}+\mu|\boldsymbol{p}| \cdot|\boldsymbol{q}| \cdot \cos \angle(\boldsymbol{p}, \boldsymbol{q}) \Leftrightarrow 7=16 \lambda+4 \frac{1}{2} \mu, \\
& \text { 2) } \boldsymbol{n} \cdot \boldsymbol{q}=\lambda \boldsymbol{p} \cdot \boldsymbol{q}+\mu \cdot|\boldsymbol{q}|^{2} \Leftrightarrow \boldsymbol{n} \cdot \boldsymbol{q}=\lambda|\boldsymbol{p}| \cdot|\boldsymbol{q}| \cdot \cos \angle(\boldsymbol{p}, \boldsymbol{q})+\mu|\boldsymbol{q}|^{2} \Leftrightarrow 3=4 \frac{1}{2} \lambda+\mu .
\end{aligned}
$$

The solution to the obtained system can be computed easily:

$$
\left\{\begin{array}{l}
16 \lambda+2 \mu=7 \\
2 \lambda+\mu=3
\end{array} \Leftrightarrow \lambda=\frac{1}{12}, \mu=\frac{17}{6} .\right.
$$


The length of the vector $\boldsymbol{n}$ can be computed as follows:

$$
|\boldsymbol{n}|^{2}=(\boldsymbol{\lambda} \boldsymbol{p}+\boldsymbol{\mu q})^{2}=\lambda^{2}|\boldsymbol{p}|^{2}+2 \lambda \boldsymbol{\mu} \boldsymbol{p} \cdot \boldsymbol{q}+\boldsymbol{\mu}^{2}|\boldsymbol{q}|^{2}=\frac{1308}{144}, \text { i.e. }|\boldsymbol{n}|=\frac{\sqrt{1308}}{12} .
$$

The angle between the vectors $\boldsymbol{n}$ and $\boldsymbol{p}$ is computed using the definition of scalar product of vectors:

$$
\cos \angle(\boldsymbol{n}, \boldsymbol{p})=\frac{\boldsymbol{n} \cdot \boldsymbol{p}}{|\boldsymbol{n}| \cdot|\boldsymbol{p}|}=\frac{7 \cdot 12}{\sqrt{1308} \cdot 4}=\frac{7 \sqrt{1308}}{436} \approx 0,58 \text {. }
$$

Note that in this problem there are no issues with the value of the cosine (it is in the range of values). Students' biggest problem in this situation is the understanding of the concept of linear dependence of vectors and its connection to coplanar vectors, i.e. the relations between the algebraic notions with geometric representations. Having in mind that linear dependence of vectors is studied not only in the VA lecture notes, but in the elective course of Linear algebra and analytic geometry in the III year of secondary education as well (19 students), the results show that students failed either the first stage of remembering (11 students) or the second stage of understanding.

If the problem is formulated differently, namely if instead of the notion of coplanar vectors, we set the formulation through a linear combination (we will refer to it as Modified Problem 2), then most of the students come to its algebraic solution just following the algebraic pattern. Justification might be found in (Pujol 2017), where using a linear combination of vectors can be one concept of proving the existence of dot and cross product. It is not the case in our VA curriculum, but one may consider different approaches in order to bring the definitions and the connections to Linear Algebra in general closer to students.

The following problem addresses the issue of applying gained knowledge to obtain a proof of a geometry theorem that students have learned in the sixth grade of primary school.

Problem 3. Determine the angle $\varphi$ between the diagonals of the parallelogram with acute angle $\alpha$ and sides with lengths $a$ and $b$. Using the obtained result, prove that the diagonals in a rhombus are mutually orthogonal. Solution. Let $A B C D$ be a parallelogram. The idea of the solution is to set the vectors $\boldsymbol{A} \boldsymbol{B}$ and $\boldsymbol{A D}$ as such that $|\boldsymbol{A} \boldsymbol{B}|=a$ and $|\boldsymbol{A D}|=b$. Also, $\angle(\boldsymbol{A B}, \boldsymbol{A D})=\alpha$. To simplify the notation we will denote the vectors $\boldsymbol{A} \boldsymbol{B}$ and $\boldsymbol{A D}$ by $\boldsymbol{a}$ and $\boldsymbol{b}$, respectively.

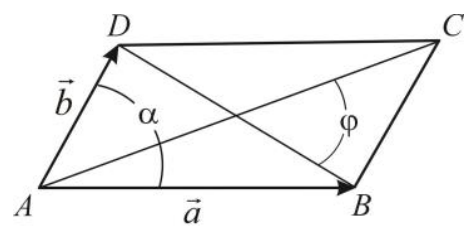

Figure 2. The Sketch Students are Expected to Draw

Denote by $\boldsymbol{d}_{1}$ and $\boldsymbol{d}_{2}$ the vectors of diagonals $\boldsymbol{A C}$ and $\boldsymbol{B D}$ of the parallelogram, respectively, and by $\varphi$ the angle between them. Clearly, $\boldsymbol{d}_{1}=\boldsymbol{a}+\boldsymbol{b}$ and $\boldsymbol{d}_{2}=\boldsymbol{a}-\boldsymbol{b}$. By the definition of scalar product of vectors, we obtain that:

$$
\cos \varphi=\frac{(\boldsymbol{a}+\boldsymbol{b})(\boldsymbol{a}-\boldsymbol{b})}{|\boldsymbol{a}+\boldsymbol{b}| \cdot|\boldsymbol{a}-\boldsymbol{b}|}=\frac{\boldsymbol{a}^{2}-\boldsymbol{b}^{2}}{|\boldsymbol{a}+\boldsymbol{b}| \cdot|\boldsymbol{a}-\boldsymbol{b}|} .
$$

Note that, $\left|\boldsymbol{d}_{1}\right|^{2}=(\boldsymbol{a}+\boldsymbol{b})^{2}$ and $\left|\boldsymbol{d}_{2}\right|^{2}=(\boldsymbol{a}-\boldsymbol{b})^{2}$. Then, by the definition of dot product, we obtain that $\left|\boldsymbol{d}_{1}\right|=\sqrt{a^{2}+2 a b \cos \alpha+b^{2}}$ and $\left|\boldsymbol{d}_{2}\right|=\sqrt{a^{2}-2 a b \cos \alpha+b^{2}}$, so the final result gets the form

$$
\begin{gathered}
\cos \varphi=\frac{a^{2}-b^{2}}{\sqrt{\left(a^{2}+b^{2}+2 a b \cos \alpha\right)\left(a^{2}+b^{2}-2 a b \cos \alpha\right)}}=\frac{a^{2}-b^{2}}{\sqrt{\left(a^{2}+b^{2}\right)^{2}-4 a^{2} b^{2} \cos ^{2} \alpha}} \text { i.e. } \\
\cos \varphi=\frac{a^{2}-b^{2}}{\sqrt{a^{4}+b^{4}-2 a^{2} b^{2} \cos 2 \alpha}} .
\end{gathered}
$$

There were issues in reaching the final conclusion. Firstly, only half of the students used sketches of a parallelogram and suitably set the vectors $\boldsymbol{A B}$ and $\boldsymbol{A D}$ to be such that $|\boldsymbol{A B}|=a$ and $|\boldsymbol{A D}|=b$. Secondly, 
even though some of the students use the fact that $a=b$ (the case of rhombus), the conclusion that $\cos \varphi=0$, thus $\varphi=90^{\circ}$ (i.e. the diagonals in a rhombus are mutually orthogonal) was not obtained. Clearly, one can formulate this problem differently: if the diagonals of a parallelogram are mutually orthogonal, then the parallelogram is a rhombus. We expected the students to come to this conclusion themselves. However, that was not the case with this group.

\section{Methodology and Results}

The case study took place at the Faculty of Natural Sciences and Mathematics in Skopje and was conducted among 30 mathematics first year students (involved in three different mathematics modules), but mainly studying the Mathematics Education module. We chose the Vector algebra course (VA) that is obligatory for all students. The VA course is organized by the Mathematics Institute with three hours of lectures each week and three hours of tutorials. The goals of the course are to help students acquire knowledge for derivation of formulas that characterize elementary-geometric relations in the Euclidean space and their appropriate use in solving problems, as well as geometric interpretation of the obtained results. In this process they are expected to develop certain mathematical skills and realize the importance of correct mathematical communication, especially when writing formal proofs that will prepare them for higher mathematics and physics courses. Among the university curriculum resources, the students use professors' lecture notes and the textbook (Ulčar 1996), mostly as a guide on what to learn, as well as two collections of problems, (Samardžiski 1991) and (Celakoski 1996), mainly used for worked examples and exercises.

As mentioned earlier, 30 students participated in the study: 23 newly enrolled students and 7 students who failed the VA course in the previous semester. In terms of secondary school background, 20 students attended the Natural Sciences and Mathematics A module, 1 student graduated the Faculty of Electrical Engineering and Information Technology, and 9 of them had acquired vocational education, mostly in economics (6 students). Most of them (93\%) had their secondary school matriculation examination project work in mathematics, physics or computer science. Surprisingly, as far as motivation goes, it seems that students that had average grades on their secondary school matriculation examination test in mathematics (mostly 4 or, in some cases, 3 ) are more motivated to learn the material and put more effort into it, instead of those who had a 5 or 2 (Grade 5 is excellent, 4-very good, 3-good, 2-satisfactory, and 1-unsatisfactory) The results of the experiment indirectly show that these students are more susceptible to accept new assessment criteria of their obtained knowledge.

The selected problems were chosen so we could determine the level at which they remember previously obtained knowledge, levels of understanding the notions and ideas behind the theory, and finally, the way that such knowledge can be applied differently (obtaining another proof).

We have divided the students' answers into the following types (see Table 2):

[T1] Complete answer is given.

[T2] Technically obtained a numerical result, but have not provided a conclusion or comment.

[T3] Getting half way through the solution.

[T4] Can use terminology and some basic properties.

[T5] Difficulties in understanding the basic concepts and properties of vectors and dot product, conclusion based on a wrong assumption.

[T6] No answer whatsoever.

Table 2. Number of Students with a Certain Type of Answer

\begin{tabular}{|l|llll|}
\hline & Problem 1 & Problem 2 & Modified Problem 2 & Problem 3 \\
\hline [T1] & 0 & 3 & 11 & 2 \\
[T2] & 7 & 3 & 0 & 4 \\
[T3] & 3 & 5 & 6 & 6 \\
[T4] & 9 & 7 & 6 & 6 \\
[T5] & 6 & 4 & 2 & 2 \\
[T6] & 5 & 8 & 5 & 10 \\
\hline
\end{tabular}

These types of answers relate to the first three stages of Bloom's taxonomy, but can be better explained by the levels of understanding, i.e. using the SOLO taxonomy (Hook, P., Gravett, C., Howard, M. \& John, E., 2014). Namely, [T1] represents relational level where students can relate notions and objects, can compare and analyze the obtained results, and draw conclusions as well; [T2] and [T3] represents the multi-structural level, [T4] 
represents uni-structural level, [T5] and [T6] represent the pre-structural level. Table 3 represents the number of students in each level of understanding using the SOLO taxonomy.

Table 3. Students with Certain Level of Understanding by SOLO Taxonomy

\begin{tabular}{|l|llll|}
\hline Levels of understanding & Problem 1 & Problem 2 & Modified Problem 2 & Problem 3 \\
\hline Pre-structural & 11 & 12 & 7 & 12 \\
Uni-structural & 9 & 7 & 6 & 6 \\
Multi-structural & 10 & 8 & 6 & 10 \\
Relational & 0 & 3 & 11 & 2 \\
\hline
\end{tabular}

It is obvious from Figure 3 that one third of the students have difficulties understanding the basic notions of vector algebra and are expected to repeat the VA course again. Some of the students are genuinely unable to understand the material at a deeper level, since their background knowledge is not on the required level. Some of them complain about insufficient time, since they have a higher workload on some of the other courses. Clearly, these students have to make significant changes in the way they learn the material. For instance: regularly following the professor's lectures and the teaching assistant's guidance, communicating with each other as well as with the teachers and not only communicating about which problems were given on the past exams, but about how well they understand the theoretical part and how to apply it in certain types of problems.

The groups of students whose understanding of the given subject is at uni-structural and multi-structural levels are smaller, but not significantly. Some of them must focus on better communication with each other and forming their own support groups, since their communication with the teaching assistant is commendable.

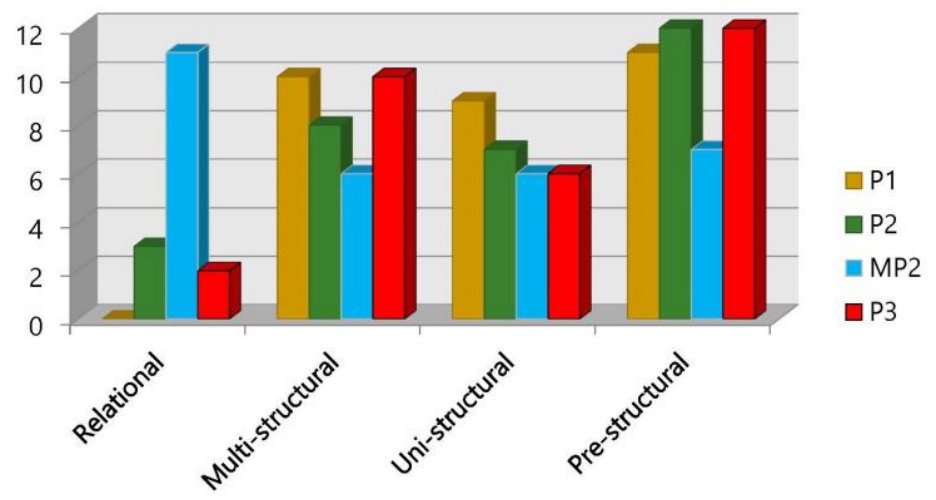

Figure 3. Interpretation of the Results from Table 3

After conducting an interview with these students, we understood that they use additional materials, such as past exams, as well as video lectures and online texts available on the Internet. It seems that students are used to avoiding the support that their professor and teaching assistant are providing, and consult them about the material just a couple of days before the exam. Almost the same could be said of those few students that qualify for the relational level of understanding. We have discovered that most of these students are under pressure to obtain a higher grade, since they want to qualify for scholarship from the Ministry of Education and Science. Their focus is therefore misplaced. We suspect that in these cases that is the reason why their performance is not on the highest level. These students should be encouraged to create positive feelings about learning or solving exercise problems, such as challenge, interest, competition and a sense of significance and achievement.

\section{Discussion}

The teaching and learning in universities is currently quite traditional: the professor covers the course material through lectures, while the teaching assistant conducts the exercises. We investigated our observations of insufficient critical reasoning by testing students with straightforward problems from Vector algebra course curriculum material. The course material is connected with background knowledge of trigonometry, logic and geometry from secondary school. We expected that the students had a well-structured knowledge base. Clearly, that was not the case. Although it is a general opinion that "mathematics cannot be learned with memorization", it is evident that memorizing is necessary to some extent: the basic notions need to be memorized and connected with some basic examples and counterexamples that can lead to understanding the essence of that notion. 
The results show that when problems are mostly numerical and correctly formulated using the terminology from vector algebra courses, one third of the students can obtain the final result (as in problem 2). It is our opinion that the students are unable to make connections between coplanar vectors and linear dependence of vectors, i.e. are unable to independently perceive the relationship between geometric representation of vectors and the algebraic notion of linear (in)dependence. This means that for two thirds of the students the level of understanding does not exceed the multi-structural level. However, more than half of the students solve such problems very successfully when they are formulated using terminology from linear algebra (modified problem 2) and come relatively easily to an algebraic solution just applying a standard scheme of solving, without any deep reasoning and without a need of creating a geometric representation. One explanation for such results is students' education and experience. Namely, in the secondary school curriculum of the elective course Linear algebra and analytic geometry ( $3^{\text {rd }}$ year of high school, i.e. junior high school), students were instructed in solving problems with linear dependence of vectors just using the pattern that the definition of linear dependence requires.

The students' results are quite different when it comes to solving a numerical problem that has no solution or when it is not properly set (problem 1). Nobody has even tried to provide a conclusion (even an incorrect one) or to question why the final result is such as it is. It is clear that one third of the students have "weaknesses" in the previously obtained mathematical knowledge from secondary school (properties of trigonometric functions). Also, one third of the students were not capable of understanding the problem at all when it does not fit in any scheme and the final third were capable of using the definition and some basic properties, but have difficulties in obtaining a numerical result. Among these students, we noticed two types of misconceptions (or errors): computational and conceptual. Conceptual errors were due to students' lack of distinguishing vectors from scalars (an example can be seen in Appova, A. \& Berezovski, T., 2013) and vector from the length of a vector. These errors occur because students have misunderstood the underlying concepts of a vector and length of a vector, so later on there were problems in understanding the operation dot product. An explanation can be found in Tabaghi (2010) who suggests that students' ability to transition between operational thinking ("conceiving a mathematical entity as a product of a certain process") to structural thinking ("involves the conception of mathematical entity as an object") is critical. Appova, A. \& Berezovski, T., 2013 suggests that a problem and/or solution should "emphasize a geometric meaning", i.e. it is important for students to explore abstract concepts through geometric representations to overcome the difficulties and misconceptions.

Similarly, as in providing the solution in problem 1, students had difficulties in reaching the final conclusion in problem 3. Only two out of thirty students had solved the problem correctly and $10 \mathrm{did}$ not even try to solve it. It was noticeable that two-thirds of the students had a geometric representation of the problem (a rhombus was drawn and the suitable vectors were noted). Among the rest of the students, either conceptual errors occur (not distinguishing vector from the length of a vector) or there was a gap in remembering a theorem learned in primary school (the diagonals in any rhombus are mutually orthogonal), that could lead them to the proper conclusion and final solution.

Learning is a complex phenomenon, and rather than trying to explain what it is, one must simply recognize its complexity and say that it is better projected in the performance of learners (Ohlsson, S. \& Rees, E., 1991). What should teachers do to increase the effectiveness of teaching? Obviously, the lectures should be less traditional: the theoretical part should not just be presented, but restructured so that existing knowledge is connected with the new knowledge (Biggs, J.B. \& Tang, C.S, 2011). The planning of the course (both lectures and exercises) should be less traditional as well: appropriate homework according to the available time of the student should be given; appropriate problems according to the abilities of the students should be proposed and the professor should facilitate the coordination of different disciplines (in the case of dot product with physics) to achieve integrated education (Aada K., 2020). This approach will help the students not only "just memorize" the new notions and/or results, but "discover" them themselves. Namely, some simple properties of vectors when dot product is in question can be "discovered" by solving concrete problems in Euclidian two-dimensional or three-dimensional space (Celakoski, 1996). The students have to be encouraged to formulate the properties themselves and to provide their proofs (as a homework assignment that can be conducted individually or in groups). In that way students boost their self-confidence and are motivated to accomplish more difficult tasks. Greater attention should be given to providing feedback on homework or tests, with constructive use of the errors, especially for conceptual errors that are the most difficult for students to recognize. This means that students must feel free to admit an error without having to worry that the admission will be used in their final grade. "Errors are important learning opportunities, but formative feedback is essential in learning from error. In the course of learning, students inevitably create misconceptions that need to be corrected so that any misunderstandings can be set right, literally in the formative stage" (Biggs, J.B. \& Tang, C.S, 2011). 
In our opinion, planning the course differently so that students become aware of different approaches and applications in sciences will contribute to a greater understanding of the course material. Future work should focus on problems in understanding other vector algebra operations (cross product and mixed product). For more certain conclusions on the students' methods of problem solving, the process of obtaining a solution should be followed, i.e. using a different methodology. Participants should be asked to report on their thinking process during or after providing a solution to a presented problem.

\section{Conclusion}

In this work we describe students' understanding and application of the algebraic operation dot product of two vectors and along with that, the relations between dot product properties and the geometric representation in concrete problems. Here, we focused on a sample of the situation with critical reasoning over many broader but just as important (and ignored) parts of the educational reforms (teachers' evaluation, grade school's system, inclusiveness, etc.) The outcome of rash reforms is that the teaching profession is not understood and valued as it should be in a progressive society. Teachers have almost lost their necessary dedication to the profession since the educational system is not really stimulating the teaching profession. Mathematics teachers experience even greater pressure because of the measurable results in detail and the societal importance of that skill. However, properly educating new generations is not just a plain obligation; it should be a fulfilling and pleasant duty to perform. Successfully teaching math means being passionate about mathematics and teaching. If students cannot learn this, then they cannot perform as good teachers. In order to achieve the best results from the learnt material and also in teaching, stimulating good communication between students is critical, as well as amongst students and teachers.

\section{References}

Aada, K. (2020). Insight on Planning and Assessing the Teaching-Learning Process, International Journal on Social and Education Sciences, 2(2), 88 - 96.

Alcantara, E. C. \& Bacsa, J. M. P. (2017). Critical Thinking and Problem Solving Skills in Mathematics of Grade-7 Public Secondary Students, Asia Pacific Journal of Multidisciplinary Research, 5(4)

Anderson, L.W., (Ed.), Krathwohl, D.R., (Ed.), Airasian, P.W., Cruikshank, K.A., Mayer, R. E., Pintrich, P.R., Raths, J. \& Wittrock, M.C. (2001). A taxonomy for learning, teaching, and assessing: A revision of Bloom's Taxonomy of Educational Objectives (Complete edition), New York: Longman.

Appova, A. \& Berezovski, T. (2013). Commonly Identified Students' Misconceptions about Vectors and Vector Operations, In Brown, S., Karakok, G., Hah Roh, G. \& Oehrtman, M. (Eds.), Conference on research in undergraduate mathematics education: Crume xvi (Vol. 2, pp. 8-17). Denver: RUME.

Biggs, J.B. \& Collis, K.F. (1982). Evaluating the quality of learning: the SOLO taxonomy (structure of the observed learning outcome), Educational psychology series, New York: Academic Press.

Biggs, J.B. \& Tang, C.S. (2011). Teaching for quality learning at university: what the student does (4th ed.). Maidenhead: McGraw-Hill; Society for Research into Higher Education; Open University Press.

Bloom, B.S. (Ed.), Engelhart, M.D., Furst, E.J., Hill, W.H. \& Krathwohl, D.R. (1956). Taxonomy of educational objectives: The classification of educational goals, Handbook 1: Cognitive domain, New York: David McKay.

Celakoski, N. (1996). Problems in linear algebra, (Fourth edition, Prosvetno delo, Skopje) (in Macedonian).

Jacob, S. M. (2012). Mathematical achievement and critical thinking skills in asynchronous discussion forums, Procedia - Social and Behavioral Sciences, 31, 800-804, doi.org/10.1016/j.sbspro.2011.12.144

Harel, G. \& Sowder, L. (2005). Advanced Mathematical Thinking at Any Age: Its Nature and Its Development, Mathematical Thinking and Learning, 7 (1), 27 - 50.

Hook, P., Gravett, C., Howard, M. \& John, E. (2014). SOLO Taxonomy in Maths. Strategies for thinking like a mathematician. Essential Resources Educational Publishers Limited, New Zealand.

Krathwohl, D.R. (2002). A Revision of Bloom's Taxonomy: An Overview, Theory into practice, 41 (4), 212 218.

Lai, E. R. (2011). Critical thinking: a literature review, Research report, Pearson.

Ohlsson, S. \& Rees, E. (1991). The function of conceptual understanding in the learning of arithmetic procedures, Cognition and Instruction, 8(2), 103 - 179.

Pujol, J. (2017). The linear combination of vectors implies existence of cross and dot products, Interantional Journal of Mathematical Education in Science and Technology, 1-15, doi: 10.1080/0020739X.2017.1408149 
Samardžiski, A. (1991). Problems in vector algebra, (Ss. Cyril and Methodius University, Skopje) (in Macedonian)

Sumarna. N., Wahyudin \& Herman, T. (2017). The Increase of Critical Thinking Skills through Mathematical Investigation Approach, IOP Conf. Series: Journal of Physics: Conf. Series 812012067 doi:10.1088/1742-6596/812/1/012067

Tabaghi, S. G. (2010). The use of DGS to support students' concept image formation of linear transformation. Frosnan, P. Erchick, D. B., \& Flevares, L. (Eds.) Proceedings of the 32nd annual meeting of the North American Chapter of the International Group for the Psychology of Mathematics Education. Columbus, $\mathrm{OH}$; The Ohio State University.

Ulčar, J. (1996) Analytic geometry, (Second edition, Numerus Library, Skopje) (in Macedonian)

White, H. S. (1906). How should the college teach analytic geometry, Bull. Amer. Math. Soc., 12 (10), $493-498$.

Zapalska, A.M., Nowduri, S., Imbriale, P., Woroblewski, B. \& Glinski, M. (2018). Framework for Critical Thinking Skills Development Across the Business Curriculum Using the $21^{\text {st }}$ Century Bloom's Taxonomy, Interdisciplinary Education and Psychology, 2(2):2. 